\title{
Control of Subcritical Thermal Power Units Based on Grid Primary Frequency
}

\author{
Jun $\mathrm{Li}^{1,}$, , Huicong $\mathrm{Li}^{2, \mathrm{~b}}$, Zhaoxin Wang ${ }^{2, \mathrm{c}}$ and Mingjun $\mathrm{Xu}^{3, \mathrm{~d}}$ \\ ${ }^{1}$ Shandong Electric Power Research Institute, Jinan 250002, China \\ ${ }^{2}$ Dispatching \& Control Center, Shandong Electric Power Corporation, Jinan 250001, China \\ ${ }^{3}$ Huaneng Weihai Power Generation Co., Ltd., Weihai 264205, China \\ alijun_sdu@hotmail.com, ${ }^{b}$ Ihc_111@163.com, 'wzx19831222@163.com, djmx7217@sohu.com
}

Keywords: power grid, primary frequency compensation, thermal power units, air-fuel control Abstract. New energy connected to power grid brings new challenges to optimal operation of power systems. The transient stability of power grid mainly depends on primary frequency compensation of thermal power units. By analyzing the theory and practice of DEH and coordinated control system(CCS) of thermal power units, combined with the insufficient of actual primary frequency compensation strategy response to frequency of accidents, the optimization of primary frequency compensation of subcritical thermal power unit's frequency control and fuel-air control were offered. The practical application results show that the proposed control strategy can effectively control the subcritical thermal power units to achieve the stability of power grid primary frequency.

\section{Introduction}

As is well known, power grid frequency is one of the three indicators of power quality, it reflects the balance between the generation of active power and load, and it is an important control parameter of the power system operation. The safety and efficiency of the majority of users of electrical equipment and power equipment have a close relationship with power grid frequency. Equipment of users is generally driven by the motor, the same as equipment of power plant. The frequency fluctuation has a critical influence over them. With the development of science and technology, some new electronic equipment and precision processing equipment put forward higher request to power grid frequency, the frequency fluctuations will lead to lower product quality or equipment damage. On the IEEE 446-1995 standard and BS EN50160:1995 standard, $\pm 0.5 \mathrm{~Hz}$ is the many devices' maximum tolerance of the frequency fluctuations. The occurrence of unexpected failures in the power grid will break the balance between the generation of active power and load. With the development of the power system, single device failures bring a growing loss of generating power grid. Only relying on manually adjust the power generation output will require a longer time to achieve a new balance. To solve these problems, the way is only one that is the techniques of primary frequency compensation and automatic generation control. It's a good idea to use different logical controls according to its own regulation performance, to maintain the balance of power system supply and demand automatically, thus ensuring the quality of the power system frequency.

Although the new and effective theories and design methodologies being continually developed in the automatic control field, Proportional-Integral-Derivative (PID) controllers are still by far the most widely adopted controllers in industry owing to the advantageous cost/benefit ratio they are able to provide. In fact, although they are relatively simple to use, they are able to provide a satisfactory performance in many process control tasks. A thermal power is normally controlled by multi-loop PI/PID controllers. The control performance of these loops is adversely affected by inter-loop interactions. In addition, normal working of a power plant is severely affected by the occurrence of a range of system disturbances. Being a highly coupled system, the disturbances in one part of the plant can have a significant effect on the rest of the plant as well. In order to minimize the influence of both plant-wide interactions and disturbances so as to ensure a higher rate of load change without violating thermal constraints, a coordinated control strategy is required. 


\section{Unit Control System's Structure}

The unit load controller essentially maintains the balance between thermal power in the boiler, and mechanical-electrical power developed by the turbine generator. Fundamental to this balance is the steam pressure at the inlet to the throttle valves (TVs) or turbine governor valves. There are many ways in which this can be done, but increasingly coordinated or integrated controls are used as shown in Fig.1.

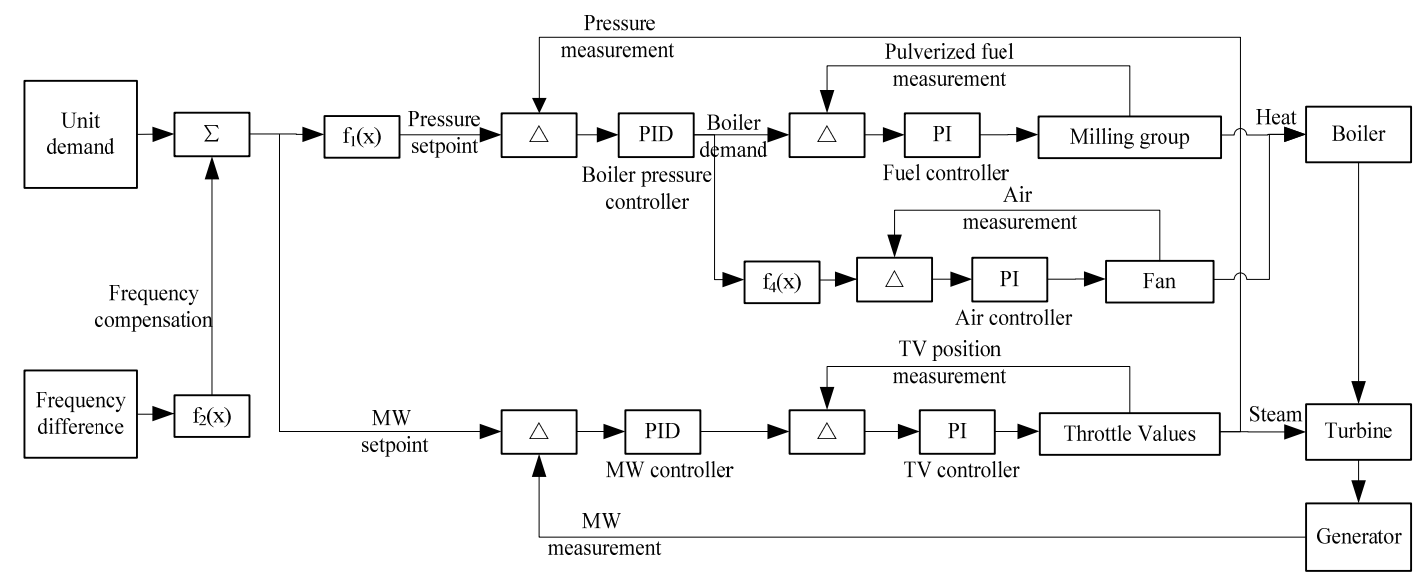

Fig.1. Unit fuel and MW controllers of power plant

In this figure the controlled outputs are steam pressure and MW load, and the controlled variables are fuel, air etc. and TV position. In operation the unit demand sets the set-point for pressure and power output, either locally or remotely from the load dispatch centre, and the control systems do the rest. With most plants now controlled by distributed control systems, it is fairly straightforward to set controller parameter values for stable operation over an acceptable load range. There are some key issues that must be discussed in relationship to Fig. 1. The most important issues are the control of fuel and air control. In practice, it is not possible to measure the pulverized fuel feedback and in addition the milling block is not just one mill but many mills. For example, it is up to six for a 660MW unit. It is important to mills and fans that their capacity must fit to the requirement of unit load. Fuel and air ratio control is very important to the main steam pressure and temperature keeps stably. At the same time, how to effectively ensure coal-air-water appropriate action when the primary frequency action, is the key to reduce the unit fluctuations and ensure the safe operation of the unit.

\section{Selector Control}

Selector control can be viewed as the inverse of split range control. In split range there is one measured signal and several actuators. In selector control there are many measured signals and only one actuator. A selector is a static device with many inputs and one output.

There are two types of selectors: maximum and minimum. For a maximum selector the output is the largest of the input signals. There are situations where there are several controlled process variables that must be taken into account. One variable is the primary controlled variable, but it is also required that other process variables remain within given ranges. Selector control can be used to achieve this. The idea is to use several controllers and to have a selector that chooses the controller that is most appropriate. One example of use is where the primary controlled variable is temperature and we must ensure that pressure does not exceed a certain range for safety reasons.

The principle of selector control is illustrated in Fig.2. The primary controlled variable is the process output $y$. There is an auxiliary measured variable $z$ that should be kept within the limits $z_{\min }$ and $\mathrm{z}_{\max }$. The primary controller $\mathrm{C}$ has process variable $\mathrm{y}$, setpoint $\mathrm{y}_{\mathrm{sp}}$, and output $\mathrm{u}_{\mathrm{N}}$. There are also secondary controllers with measured process variables that are the auxiliary variable $\mathrm{z}$ and with setpoint that are bounds of the variable $z$. The outputs of these controllers are $u_{H}$ and $u_{L}$. The controller $\mathrm{C}$ is an ordinary PI or PID controller that gives good control under normal circumstances. The output of the minimum selector is the smallest of the input signals; the output of the maximum selector is the largest of the inputs. 


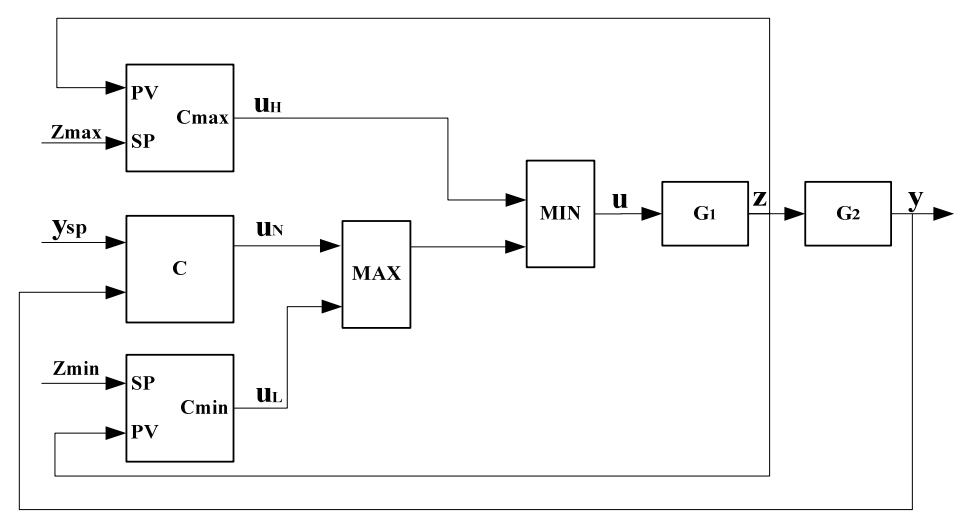

Fig.2. Selector control

Under normal circumstances the auxiliary variable is larger than the minimum value $z_{\min }$ and smaller than the maximum value $z_{\max }$. This means that the output $u_{H}$ is large and the output $u_{L}$ is small. The maximum selector, therefore, selects $u_{N}$ and the minimum selector also selects $u_{N}$. The system acts as if the maximum and minimum controller were not present. If the variable $\mathrm{z}$ reaches its upper limit, the variable $\mathrm{u}_{\mathrm{H}}$, becomes small and is selected by the minimum selector. This means that the control system now attempts to control the variable $\mathrm{z}$ and drive it towards its limit. A similar situation occurs if the variable $\mathrm{z}$ becomes smaller than $\mathrm{z}_{\min }$. In a system with selectors, only one control loop at a time is in operation. The controllers can be tuned in the same way as single-loop controllers. Selector control is very common in order to guarantee that variables remain within constraints. The technique is commonly used in the power industry for control in boilers and power systems.

\section{Industrial Application}

For example, in one 330MW subcritical coal-fired power plant, its rated speed is 3000rpm, speed governor droop is $5 \%$, and power compensation quantity is $26.4 \mathrm{MW}$. As we known, primary air flow and temperature are significant influences in mill control. Secondary air flow is important in the furnace but does not affect the mill. Here we focus on the secondary air flow control issues.

Fuel-air control. As shown in Fig.1, the fuel-air control adopt ratio control, it has disadvantage. When the unit demand is increased, the boiler demand is increased; there may be lack of air because the setpoint of the air controller increases first when the dual controller has increased the fuel flow. The system cannot compensate for perturbations in the air channel. A much improved system uses selectors, such as is shown in Fig.3.

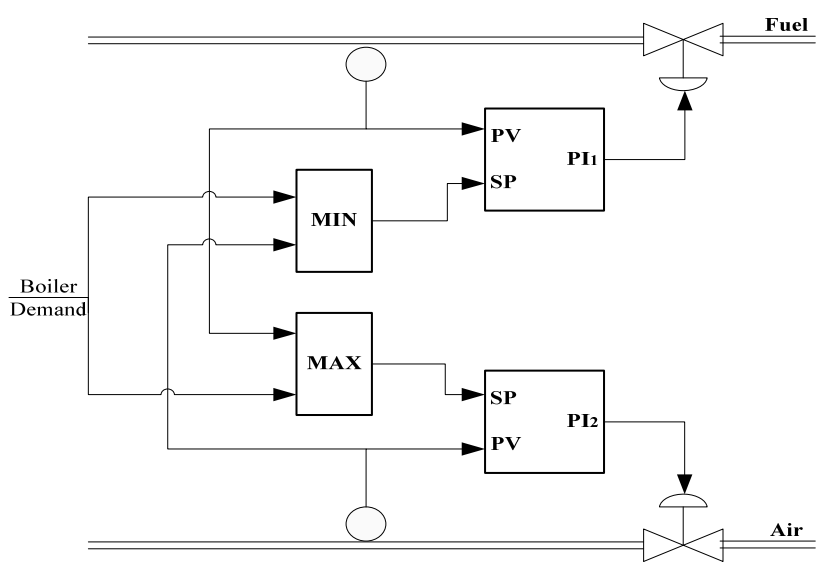

Fig.3. Fuel-air controller based on selectors

The system uses one minimum and one maximum selector. There is one PI controller for fuel flow and one PI controller for the air flow. The setpoint for the air controller is the larger of the command signal and the fuel flow. This means that the air flow will increase as soon as more energy is demanded. Similarly; the setpoint to the fuel flow is the smaller of the demand signal and the air flow. 
This means that when demand is decreased, the setpoint to the dual flow controller will immediately be decreased, but the setpoint to the air controller will remain high until the oil flow has actually decreased. The system thus ensures that there will always be an excess of air. It is important to maintain good air quality. It is particularly important in ship boilers because captains may pay heavy penalties if there are smoke puffs coming out of the stacks when in port.

Primary Frequency control of CCS. When the unit primary frequency action amplitude is small, that is, the amount of compensation $\Delta \mathrm{P}$ of primary frequency within a certain amplitude range, using the boiler heat storage to realize the change of load demand, this time the main steam pressure substantially no change or a small change, boiler master may not regulate or slow regulate. If the unit load is in quick adjustment stage, the boiler master must quickly adjust, to regulate the air, coal etc. quickly, to ensure the stability of the main steam pressure and other parameters to ensure that the needs of load control. Primary frequency control of CCS is shown as Fig.4.

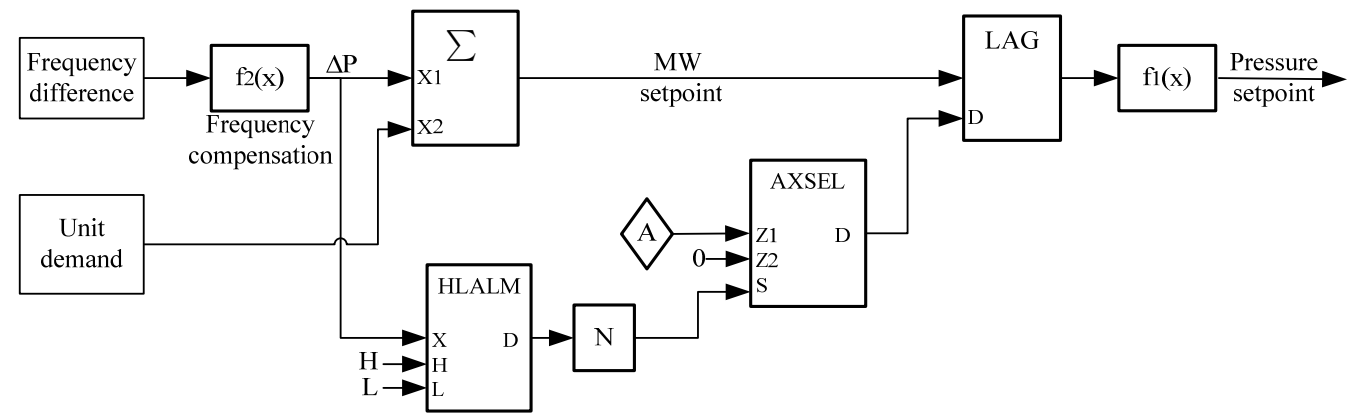

Fig.4. Primary frequency control of CCS

Wherein, AXSEL is analog selector module, HLALM is high and low limit alarm module. Described in the module of one-order inertial LAG formula expressed as

$$
C(s)=\frac{K}{T s+1}
$$

The value $K$ is 1 , the value of $T$ is the input value of input port $D$, which is output of the selector.

\section{Conclusions}

By analyzing the DEH and CCS control of the subcritical thermal power unit, combined with the actual power load frequency control strategy to deal with the deficiency of the frequency accidents, optimization of PFC logic is put forward. The practical application results show that the proposed control strategy can effectively control subcritical thermal power unit to realize the stability of the power system frequency. It is benefit to intelligent power grid construction.

\section{References}

[1] Karl J.Astrom, and Tore Hagglund, Advanced PID Control, ISA, (2006)70-73.

[2] D.R.Coyghanowr, Process Systems Analysis and Control, McGraw-Hill, New York, 1991.

[3] Harris, T.J. "Assessment of closed loop performance", Canadian Journal of Chemical Engineering, 67, 856-861, 1989.

[4] M. Zhuang, D.P. Atherton, Automatic tuning of optimum PID controllers, IEE ProcD, 140, 1993, pp. 216-224.

[5] F.Shinskey, Process Control Systems Application Design Adjustment, McGraw-Hill, New York, 1996. 